Miguel V. Crisostomo Jr., MD

Celso V. Ureta, MD

Department of Otorhinolaryngology

Head and Neck Surgery

Veterans Memorial Medical Center
Correspondence: Dr. Celso V. Ureta Department of Otorhinolaryngology Head and Neck Surgery

Veterans Memorial Medical Center

North Avenue, Diliman Quezon City 1104

Philippines

Phone: (632) 9276426 loc 1359

Email: enthns_vmmc@yahoo.com

The authors declared that this represents original material that is not being considered for publication or has not been published or accepted for publication elsewhere, in full or in part, in print or electronic media; that the manuscript has been read and approved by the authors, that the requirements for authorship have been met by the authors, and that the authors believe that the manuscript represent honest work.

Disclosures: The authors signed a disclosure that there are no financial or other (including personal) relationships, intellectual passion, political views or beliefs, and institutional affiliations that might lead to conflict of interest.

\section{Autologous Tracheal Cartilage Composite Graft for a Subglottic Defect after Laryngotracheal Resection for Invasive Papillary Thyroid Carcinoma}

\section{ABSTRACT}

Objective: To present a function-preserving surgical technique of post-laryngotracheal resection reconstruction of the subglottic airway using autologous tracheal cartilage composite graft.

\section{Methods:}

$\begin{array}{ll}\text { Design: } & \text { Case Report } \\ \text { Setting: } & \text { Tertiary Government Training Hospital } \\ \text { Participants: } & \text { One }\end{array}$

Results: A 77-year-old woman diagnosed with papillary thyroid carcinoma with laryngotracheal invasion underwent total thyroidectomy with laryngotracheal resection. The tracheal defect was reconstructed using end-to-end anastomosis of the trachea to the remaining cricoid. The cricoid (subglottic) defect was repaired using the harvested tracheal cartilage with mucosa. Postoperatively, the patient was maintained on nasogastric tube feeding and tracheostomy tube for 2 weeks. Subsequently, the nasogastric tube and tracheostomy tube were removed and the patient tolerated oral feeding without any airway problem. The last follow-up of the patient was 6 months post-operatively without complications.

Conclusion: Autologous tracheal cartilage may be a potentially promising composite graft for reconstruction of the cricoid (subglottic) defect in a patient following laryngotracheal resection for invasive papillary thyroid carcinoma of the larynx and trachea.

Keywords: tracheal composite graft; laryngotracheal resection; crico-tracheal anastomosis; papillary thyroid carcinoma; subglottic defect

Laryngotracheal invasion occurs in 3.6 to $22.9 \%$ of patients with thyroid cancer and is the most common site of local invasion following the strap muscles and the recurrent laryngeal nerve. ${ }^{1}$ The most effective treatment for thyroid cancer invading into the larynx and trachea is complete surgical resection of the tumor. Surgical options in this situation include the shave procedure, partial laryngectomy and total laryngectomy. ${ }^{2}$ Intraluminal extension requires resection of the tracheal wall and a portion of the larynx including the cricotracheal cartilage. ${ }^{2}$ Several 


\section{SURGICAL INNOVATIONS AND INSTRUMENTATION}

surgical techniques which aim to avoid postoperative deformity of the airway and subglottic stenosis utilizing a composite nasal cartilage graft, an auricular cartilage graft and a costal cartilage graft have all been used as supporting material to repair the defect and reconstruct the airway. ${ }^{1}$

However, to the best of our knowledge based on a search of HERDIN, MEDLINE (PubMed) and Google Scholar using the search terms "composite tracheal graft," "subglottic defect," "invasive papillary thyroid carcinoma," and "laryngotracheal resection," a function-preserving surgical method of resection and reconstruction for thyroid cancer invading into the intra-lumen of cricotracheal region has not yet been established.

It is the objective of this study to present a surgical technique of cricoid (subglottic) reconstruction using an autologous tracheal composite graft after laryngotracheal resection in a patient with invasive papillary thyroid cancer.

\section{CASE REPORT}

A 77-year-old woman consulted due to anterior neck mass and recurrent hemoptysis. The neck mass was noted 35 years prior to consult. A neck computed tomography with contrast revealed a $2.6 \times 2.5$ $x 2.4 \mathrm{~cm}$ heterogeneous enhancing mass in the region of the left thyroid lobe with extension to the trachea causing narrowing at the level C7T1. (Figure 1A) A fine needle aspiration biopsy report was consistent with papillary thyroid carcinoma. Indirect laryngoscopy revealed a left paralyzed true vocal cord with adequate airway, confirmed by flexible nasopharyngolaryngoscopy. (Figure 1B) The rest of the ENT examination was unremarkable.

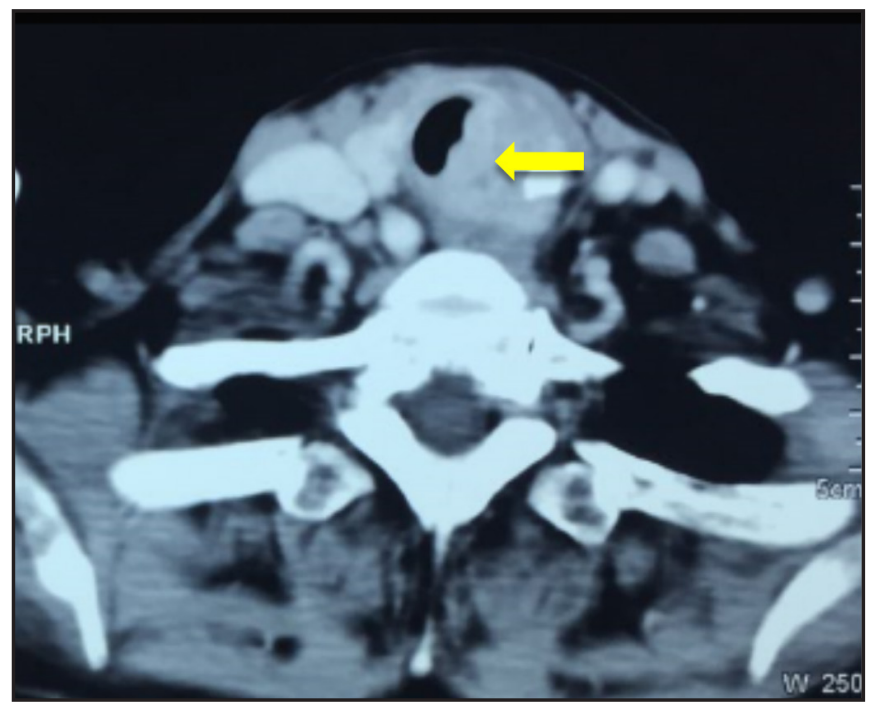

Figure 1A. Preoperative Computed Tomography (axial image at the level of the cricoid) confirming intraluminal extension of the thyroid mass (arrow).

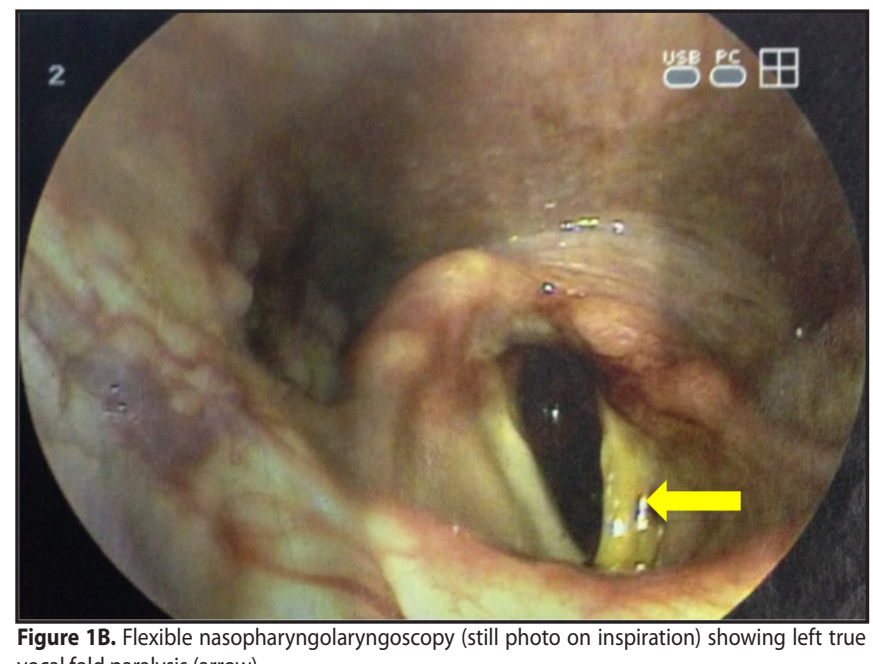

vocal fold paralysis (arrow)

The patient underwent total thyroidectomy with laryngotracheal resection followed by cricotracheal anastomosis and reconstruction of the subglottic airway using autologous tracheal composite graft. We describe our surgical technique.

\section{Surgical Technique}

1. Total Thyroidectomy: Right thyroid lobectomy and isthmusectomy were done with preservation of the right recurrent laryngeal nerve. The left lobe was totally occupied by a hard mass of approximately $3.0 \times 2.0 \mathrm{~cm}$. The thyroid mass was noted to be adherent to the left inferolateral thyroid cartilage, left lateral aspect of the cricoid and the first three (3) tracheal rings including the left recurrent laryngeal nerve. The left thyroid tumor was shaved off from the anterior surface of the left trachea, cricoid and thyroid cartilage exposing the trachea, cricoid and thyroid cartilage.

2. Laryngotracheal Resection: After identifying the tumor invasion of the trachea and larynx, the involved trachea, cricoid and thyroid cartilage were resected with adequate gross tumor margins. The surgical specimen included part of the left ala of the thyroid cartilage exposing the paraglottic muscles, left anterolateral part of the cricoid (subglottic area) and left anterolateral part of the first four tracheal cartilages and the resulting defect was measured using a Castroviejo caliper. (Figure 2)

3. Mobilization of the Trachea and Tracheostomy: The trachea was mobilized and an approximate tracheostomy site was identified and done to facilitate reconstruction. (Figure 3)

4. Harvesting of Tracheal Composite Graft: To facilitate reconstruction using end-to-end anastomosis of the trachea to the cricoid, the first 4 remaining portions of tracheal cartilage 


\section{SURGICAL INNOVATIONS AND INSTRUMENTATION}

Philippine Journal Of Otolaryngology-Head And Neck Surgery

Vol. 34 No. 1 JANUARY - JUNE 2019

PIOHIS
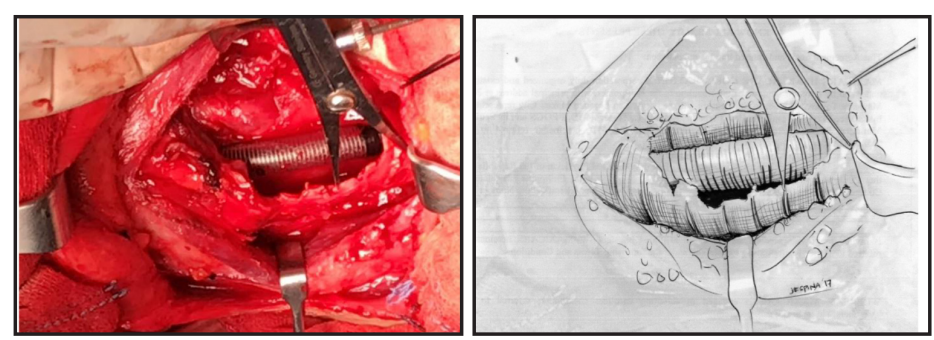

Figure 2. Laryngotracheal resection and harvesting of tracheal composite graft. (A Castroviejo caliper is used to measure tracheal graft).
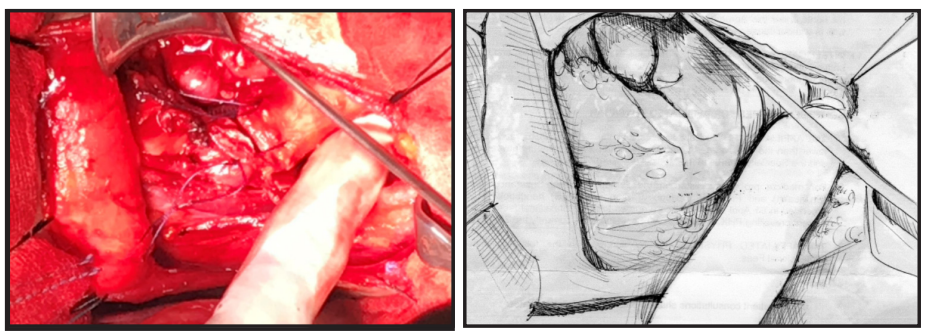

Figure 3. Mobilization and anchoring of the trachea and placement of tracheostomy tube.
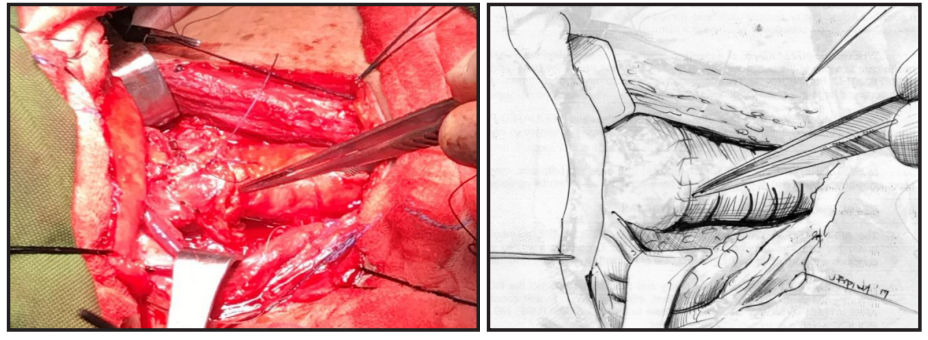

Figure 4. Tracheo-cricoid fixation. (Note the trachea was approximated to the remaining cricoid using vicryl 2-0 sutures)
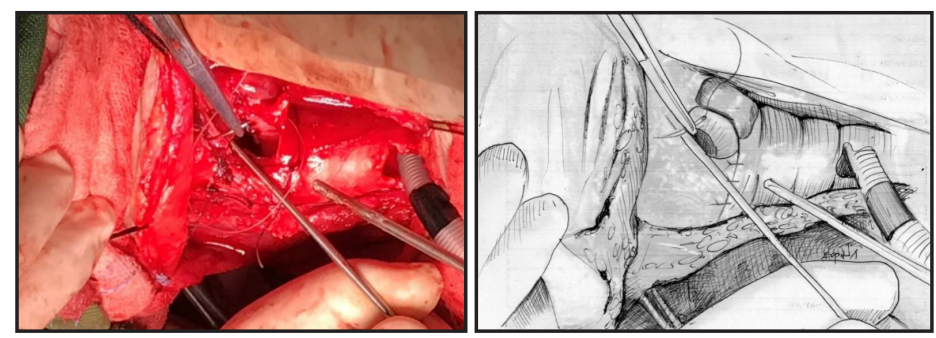

Figure 5. Reconstruction of the cricoid (subglottic defect). Note the harvested tracheal cartilage with mucosa (composite graft) was now fashioned to approximate the lumen of the cricoid airway.

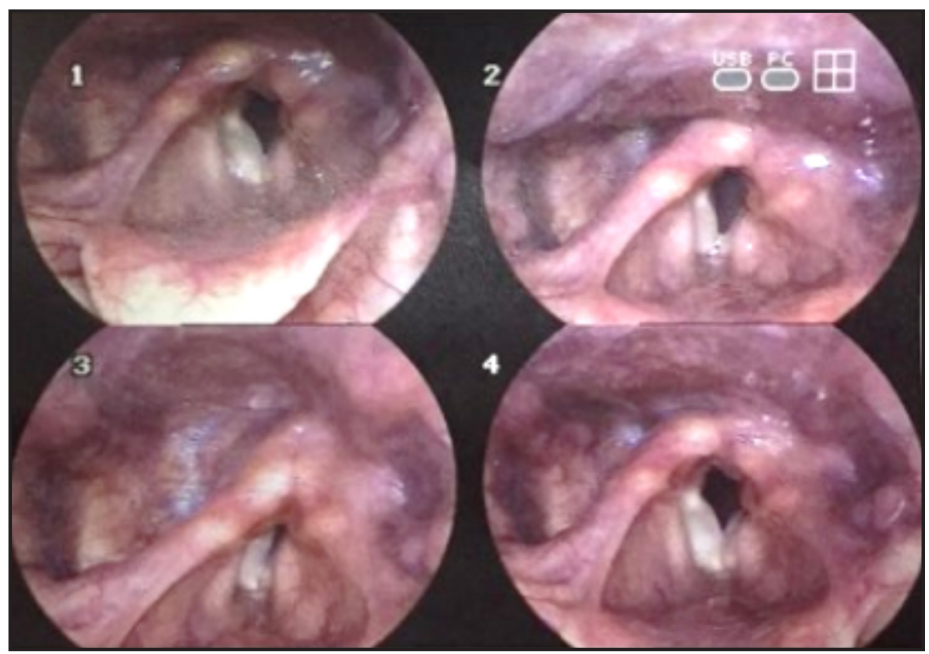

Figure 6. Flexible nasopharyngolaryngoscopy 6 months post-operatively. Note the laryngeal inlet at 1. beginning inspiration; 2. inspiration; 3 . Phonation; 4. beginning inspiration.

were resected on the right side. These tracheal cartilages were preserved, harvested and used as a composite cartilage graft for reconstruction of the cricoid cartilage (subglottic) defect. (Figure 2)

\section{Reconstruction of the Laryngotracheal Defect}

A. Tracheo-cricoid Fixation: The trachea was approximated to the remaining cricoid using vicryl 2-0 sutures at the posterior tracheal wall. The mucosa was approximated using vicryl 3-0 sutures. (Figure 4)

\section{B. Reconstruction of the Cricoid (Subglottic) Defect using Composite Tracheal Graft:}

The harvested tracheal cartilage with mucosa (composite graft) was now fashioned to approximate the lumen of the cricoid airway. The first layer was the mucosal repair using vicryl 4-0 sutures and the second layer was the side-to-side anastomosis of the cricoid to the tracheal cartilage using vicryl 3-0 monofilament suture. (Figure 5)

6. Anchoring of the Trachea: In order to prevent tension at the crico-tracheal anastomosis, the mobilized trachea was anchored to the suprasternal periosteum and sternocleidomastoid muscles on both sides to serve as an antigravity suture.

7. Closing of Incision: The strap muscles were approximated the midline. The skin incision was closed in layers.

The patient was kept on strict nihil per os (NPO) and osteorized feedings were given via nasogastric tube (NGT) for 2 weeks. Postoperatively, cefuroxime $750 \mathrm{mg}$ was given by NGT every 8 hours and with daily wound dressings for 7 days. The tracheostomy tube was 


\section{SURGICAL INNOVATIONS AND INSTRUMENTATION}

removed after 2 weeks without complications. The patient underwent radioactive iodine therapy after one month and was asymptomatic 6 months post-operatively. Endoscopy showed adequate airway with no evidence of airway deformity, subglottic stenosis, or post-operative local recurrence. (Figure 6)

\section{DISCUSSION}

Surgery remains the mainstay of therapy for locally advanced thyroid cancer with complete resection and negative margins a fundamental goal. ${ }^{2}$ It is therefore imperative that in the presence of laryngo-tracheal invasion, wide excision of the laryngo-tracheal tumor with good tumor margins be performed to ensure long-term survival. Although there is no consensus on extent of surgery when the airway is invaded by thyroid carcinoma, successful treatment requires radical excision of the tumor. However, the resection of critical structures such as trachea, cricoid and thyroid cartilage is associated with significant morbidity. ${ }^{3}$

The post-operative defect after wide resection of the laryngotracheal tumor leads to difficult airway reconstruction. The thyroid cartilage defect can be managed with soft tissue cover from the remaining strap muscles of the neck. The tracheal defect can be reconstructed by using end-to-end anastomosis to the remaining cricoid cartilage to maintain continuity of the tracheal airway. ${ }^{4}$ However, the subglottic defect in this patient was the most difficult to manage because of the possible complication of subglottic stenosis. Even a partial defect of the cricoid cartilage can result in postoperative deformity of the airway and subglottic stenosis. ${ }^{5}$ Friedman and colleagues demonstrated that up to $50 \%$ of the external laryngeal framework could be removed with internal laryngeal preservation. Another study by Price showed that $50 \%$ of the thyroid cartilage and approximately $30 \%$ of the cricoid may be resected without complex reconstruction or tracheotomy. ${ }^{2}$ In this patient there was more than $50 \%$ of the subglottic defect that needed reconstruction after resection.

Nasal, auricular and rib cartilage have been utilized as grafting materials for laryngotracheal reconstruction. ${ }^{5}$ However, to the best of our knowledge, no consensus has been reached as to the ideal graft materials. ${ }^{6}$ Other primary reconstructive options consist of local flaps such as sternocleidomastoid muscle or myoperichondral flap.?

We presented a method of using a free autologous tracheal composite graft to simplify reconstruction of the cricoid (subglottic) defect and produce an adequate subglottic airway. The success of this procedure may have been facilitated by ready availability of the tracheal cartilage composite graft in the same surgical field for repair of the subglottic area. The use of a tracheal composite graft have shown considerable success due to its ability to provide repair.

The conventional use of other cartilage grafts which all carry a risk of insufficient size and strength of the transplanted graft was avoided in our procedure. Moreover, the tracheal cartilage composite graft was approximated to the cricoid in side-to-side layers by suturing the mucosa of the tracheal cartilage to the mucosa of the cricoid cartilage and the tracheal cartilage to the cricoid cartilage to increase chances of promoting a new blood supply to the tracheal graft.

In order to improve the success of the anastomosis of the trachea to the cricoid cartilage, a tension-free repair technique was achieved by anchoring the mobilized trachea to the suprasternal periosteum and sternocleidomastoid muscles on both sides. This technique avoids the risk of anastomotic breakdown including risk of fistula formation and mediastinal infection. ${ }^{4}$

Our experience suggests that autologous tracheal cartilage may be a potentially promising composite graft for reconstruction of the cricoid (subglottic) defect in a patient who underwent laryngotracheal resection for invasive papillary thyroid carcinoma of the larynx and trachea.

\section{REFERENCES}

1. Kobayashi M, Fukuda A, Onozuka A, Kawagoe C, Wakayama F, Harada O. Design of a new technique using a free tracheal autologous graft for reconstruction of the cricoid cartilage and trachea. Surg Today. 2006: 36(4): 316-320. DOI: 10.1007/s00595-005-3154-9; PMID: 16554987.

2. Price DL, Wong RJ, Randolph GW. Invasive thyroid cancer: management of the trachea and esophagus. Otolaryngol Clin North Am. 2008 Dec; 41(6): 1155-68. DOI: 10.1016/j.otc.2008.08.002; PMID: 19040976 PMCID: PMC2750808.

3. Chaukar DA, Prabhudesai SG, Bhambhani NL, Pathak KA, Sanghvi VD. Differentiated thyroid cancer invading the hypopharynx: A case report. Indian J Otolaryngol Head Neck Surg. $2006 \mathrm{Jan}$ 58(1): 92-4. DOI: 10.1007/BF02907755; PMID: 23120251 PMCID: PMC3450624.

4. Urken ML. Prognosis and management of invasive well-differentiated thyroid cancer Otolaryngol Clin North Am. 2010 Apr; 43(2): 301-28, viii. DOI: 10.1016/j.otc.2010.02.002. PMID: 20510716.

5. Kobayashil M. Design of a new technique using a free tracheal autologous graft for reconstruction of the cricoid cartilage and trachea. Surg Today. 2006; 36(4):316-320. DOI: 10.1007/s00595-005-3154-9; PMID: 16554987.

6. Khalid, Ayesha N and Goldenberg, David, Surgical Management of Upper Airway Stenosis. Cummings Otolaryngology Head and neck Surgery. $5^{\text {th }}$ edition. Vol 1. Philadelphia: Elsevier Mosby; 2010. p. 946

7. Piazza C, Del Bon F, Barbieri D, Grazioli P, Paderno A, Perotti P. Tracheal and crico-tracheal resection and anastomosis for malignancies involving the thyroid gland and the airway Ann Otol Rhinol Laryngol. 2016 Feb; 125(2): 97-104. DOl: 10.1177/0003489415599000; PMID: 26296930. 